\title{
Individual differences in social desirability are associated with white-matter microstructure of the external capsule
}

\author{
Milan Andrejević $^{1,2}$ (D) $\cdot$ Dar Meshi $^{1,3} \cdot$ Wouter van den Bos $^{4} \cdot$ Hauke R. Heekeren ${ }^{1,5}$
}

Published online: 6 November 2017

(C) Psychonomic Society, Inc. 2017

\begin{abstract}
Humans tend to present themselves in a positive light to gain social approval. This behavioral trait, termed social desirability, is important for various types of social success. Surprisingly, investigation into the neural underpinnings of social desirability has been limited and focused only on interindividual differences in dopamine receptor binding. These studies revealed reduced dopamine receptor binding in the striatum of individuals who are high in trait social desirability. Interestingly, high dopamine signaling has been associated with low white-matter integrity, irrespective of social desirability. Based on these findings, we hypothesized that a positive association exists between trait social desirability and the white-matter microstructure of the external capsule, which carries fibers to the striatum from the prefrontal cortex. To test this hypothesis, we collected diffusion tensor imaging data and examined the relationship between fractional anisotropy of the external capsule and participants'
\end{abstract}

Milan Andrejević and Dar Meshi contributed equally to this article.

Milan Andrejević

milan.andrejevic@unimelb.edu.au

Dar Meshi

darmeshi@gmail.com

1 Department of Education and Psychology, Freie Universität Berlin, Habelschwerdter Allee 45, 14195 Berlin, Germany

2 Melbourne School of Psychological Sciences, The University of Melbourne, Melbourne, Victoria, Australia

3 Department of Advertising and Public Relations, Michigan State University, East Lansing, MI, USA

4 Max Planck Institute for Human Development, Berlin, Germany

5 Center for Cognitive Neuroscience Berlin, Freie Universität, Berlin, Germany social desirability — our analysis revealed a positive association. As a second exploratory step, we examined the association between social desirability and white-matter microstructure throughout the whole brain. Our whole-brain analysis revealed associations within multiple major white-matter tracts, demonstrating that socially desirable behavior relies on connectivity between distributed brain regions.

Keywords Social desirability · Impression management . Self-presentation $\cdot$ Reputation $\cdot$ Diffusion tensor imaging

Humans tend to portray themselves in a generally favorable manner, and this behavioral trait is called social desirability (Holden, 2010). For example, a person with high trait social desirability would likely deny being jealous of another's good fortune because exhibiting jealousy typically yields social disapproval and could potentially tarnish one's reputation. Behaving in a socially desirable manner not only protects one's positive reputation but also contributes to it (Uziel, 2010). Social desirability is important for various types of social success, which is demonstrated by correlations between trait social desirability and the forming and maintaining of both marital relationships and friendships (Russell \& Wells, 1992; Twenge \& Im, 2007; Uziel, 2010). In contrast to social desirability's positive relationship with social success, social desirability is negatively related to psychosocial maladjustments. For example, it is well established that social desirability is negatively correlated to both criminal and suicidal tendencies (Ivanoff \& Jang, 1991; Linehan \& Nielsen, 1983; Mills \& Kroner, 2005; Miotto \& Preti, 2008; Tan \& Grace, 2008; Twenge \& Im, 2007). Social desirability is also negatively associated with the lifetime prevalence of psychiatric disorders, and because of the social benefits that come with 
high social desirability, this trait is considered a clinically relevant protective factor against suicide and a variety of psychiatric disorders (Lane, Merikangas, Schwartz, Huang, \& Prusoff, 1990; Miotto \& Preti, 2008). Surprisingly, despite the importance of this personality trait, little is known about the neural underpinnings of individual differences in social desirability.

To the best of our knowledge, neuroscientific investigation of trait social desirability has been limited to three studies: one single photon emission computed tomography (SPECT) study and two positron emission tomography (PET) studies. These studies all assessed social desirability with a questionnaire and investigated individual differences in dopamine receptor availability. In the SPECT study, social desirability was found to negatively correlate with levels of $\mathrm{D} 2$ receptor availability in the putamen across participants (Huang et al., 2006). In line with this, the PET studies found trait social desirability to negatively correlate with D2 and D3 receptor availability in the striatum across participants (Cervenka, Gustavsson, Halldin, \& Farde, 2010; Reeves et al., 2007). To note, chronic D2 and D3 receptor blockade leads to an increase in whitematter integrity (Ebdrup, Raghava, Nielsen, Rostrup, \& Glenthøj, 2015), as do reductions in extracellular dopamine due a polymorphism in the COMT gene (Thomason et al., 2010). Therefore, the above research on dopamine receptors and social desirability, along with this research on dopamine receptors and white-matter integrity, suggests a possible positive relationship between social desirability and white-matter integrity, particularly in tracts leading to and from the striatum and prefrontal cortex - the most likely tract to exhibit this association with trait social desirability is the external capsule, which carries fibers directly to the striatum from the prefrontal cortex (Schmahmann \& Pandya, 2009). Neurocognitive consideration of social desirability also points in the direction of the external capsule. Socially desirable behavior could be driven by the need for social approval and rely heavily on processing and learning from social rewards (Crowne \& Marlowe, 1960; Uziel, 2010). Social rewards, such as discovering that others like or think highly of you, activate a network of brain regions including the ventral striatum, ventromedial prefrontal cortex, and ventral tegmental area (Davey, Allen, Harrison, Dwyer, \& Yücel, 2010; Izuma, Saito, \& Sadato, 2008; Korn, Prehn, Park, Walter, \& Heekeren, 2012; Meshi, Morawetz, \& Heekeren, 2013; Ruff \& Fehr, 2014). Functional connectivity between these areas appears to be important for computation of rewards (D. V. Smith et al., 2014). This functional connectivity must be supported by structural connectivity, and therefore the white-matter microstructure of the external capsule is a likely candidate.

We hypothesized that the white-matter microstructure of the external capsule would positively correlate with trait social desirability across participants. In other words, people who behave in a more socially desirable manner should have greater white-matter integrity of the external capsule. To address our hypothesis, we collected diffusion tensor images of 35 healthy participants. We employed whole-brain tract-based spatial statistics (TBSS) to derive diffusion tensor indices of white-matter integrity. We then extracted the mean fractional anisotropy (FA) from within our external capsule region of interest (ROI) and correlated this with individual social desirability scores, as measured by Short Form $\mathrm{C}$ of the MarlowCrowne Social Desirability Scale (Reynolds, 1982).

We also performed a whole-brain exploratory analysis, correlating voxel-wise white-matter indices with social desirability scores. To explain, social reward processing is only one of the several types of cognition and behavior that socially desirable behavior has been theorized to involve. White-matter tracts connecting brain regions involved in these other types of cognition may therefore also demonstrate associations with trait social desirability: (1) Socially desirable behavior could also be driven by the fear of social punishments. Threat of social punishment, in particular for transgression of social norms, involves activation of the lateral orbitofrontal cortex and the right dorsolateral prefrontal cortex (Spitzer, Fischbacher, Herrnberger, Grön, \& Fehr, 2007). Reactivity to both social rewards and punishments has been proposed as a possible mechanism by which people learn to abide by social norms (Rilling \& Sanfey, 2011), which is essential for socially desirable behavior (Bou Malham \& Saucier, 2016). (2) Socially desirable behavior may require deciphering other people's thoughts, feelings, and intentions, and thus involve mentalizing. Mentalizing reliably recruits a distributed network of brain regions, including the dorsomedial prefrontal cortex, bilateral temporoparietal junction, anterior temporal lobes, inferior frontal gyri, and posterior cingulate cortex (Schurz, Radua, Aichhorn, Richlan, \& Perner, 2014). (3) Socially desirable behavior may involve control of undesirable impulses for successful impression management (Uziel, 2010). Selfcontrol for the sake of reputation has been shown to require the dorsolateral prefrontal cortex (Knoch et al., 2008). (4) Finally, socially desirable behavior may require thinking about one's own personal qualities in a self-deceiving manner (Paulhus, 1984). We are not aware of a human neuroimaging study on self-deceit, however this kind of cognition likely involves self-related processing in cortical midline structures (Northoff et al., 2006). In sum, socially desirable behavior has been theoretically linked to several cognitive processes, and these processes would require computations in most of the regions of the prefrontal cortex as well as networks connecting the prefrontal cortex with the striatum and the temporal and parietal lobes. Therefore, at this early stage of research into socially desirable behavior, we performed a whole-brain exploratory investigation of the white-matter pathways connecting the above-mentioned regions and networks. 


\section{Materials and methods}

\section{Participants}

Thirty-five participants (21 female) between 19 and 34 years of age ( $M=25.7$ years, $S D=3.7$ years) participated in this study. The experimental procedure was approved by the Ethics Committee of Freie Universität Berlin, and each participant provided written informed consent before scanning. All participants were right-handed and had no known history of psychiatric disorders. These participants were also used in another neuroimaging study concerning resting state connectivity of the brain (Meshi et al., 2016).

\section{Social desirability assessment}

Social desirability was assessed using Short Form C of the Marlow-Crowne Social Desirability Scale (Reynolds, 1982). The original 33-item Marlowe-Crowne Social Desirability Scale (Crowne \& Marlowe, 1960) is widely used; however, it lacks internal consistency reliability (Loo \& Loewen, 2004). Compared to the original scale, Short Form C (Reynolds, 1982) provides better goodness-of-fit indices (Loo \& Thorpe, 2000), as well as better internal consistency (Zook \& Sipps, 1985), and construct validity (Robinette, 1991). To note, early investigation into the full, 33-item scale showed that Marlow-Crowne items indicated that social desirability can be modeled by two factors: impression management and self-deception (Paulhus, 1984). More recent investigation on a much larger sample concluded that neither the full MarlowCrowne scale, nor the Short Form C, fit this two-factor model (Leite \& Beretvas, 2005). This lack of factor structure can be seen as a disadvantage of the scale we used, the Short Form C, because we cannot pursue investigation into the specific contributions of impression management and self-deception. The scale uses a true-false response format and consists of 13 items. Each item describes a socially approved behavior that has an improbable chance of occurrence (e.g., "I'm always willing to admit it when I make a mistake"). To test our hypothesis, we correlated the scores on this scale with whitematter microstructure.

In the current study, we operationalized social desirability as a positive, prosocial personality trait. To note, however, social desirability is often assessed specifically to address the validity threat that it poses to self-report as a non-test-related variable. In other words, social desirability is often assessed as a source of experimental measurement error, and many researchers conceptualize socially desirable behavior as a questionnaire response style, collecting social desirability specifically to correct for socially desirable responding (Uziel, 2010). However, social desirability scales were initially developed to measure a trait that gives rise to biased responding (Crowne \&
Marlowe, 1960; Edwards, 1957). Furthermore, McCrae and Costa (1983) challenged the practice of using social desirability measures to rule out intersubject variance when responding to self-report questionnaires. They compared personality reports completed by individuals and their spouses and found that correcting for social desirability in self-reported measures decreased the validity of the measures (assessed by correlations between the two reports). This decrease in correlation, which is consistently found in the literature, also indicates that instead of merely capturing a response bias, social desirability scales measure a meaningful psychological construct (i.e., a personality trait) (Borkenau \& Ostendorf, 1992; Borkenau \& Zaltauskas, 2009; Diener, Sandvik, Pavot, \& Gallagher, 1991; Pauls \& Stemmler, 2003; for a review, see Uziel, 2010). McCrae and Costa also found a substantial correlation between individual's social desirability scores (Marlowe-Crowne) and ratings of their agreeableness and conscientiousness as reported by their spouses, providing further evidence that the scale represents a true and valid measure of social desirability. Like other traits, social desirability shows internal consistency, consistency across situations, stability over time, predicts real-life behaviors (Uziel, 2010), and has meaningful, consistent associations with adaptive psychological variables across cultures (Bou Malham \& Saucier, 2016).

As mentioned above, participants were also used in another neuroimaging study concerning resting state connectivity of the brain (Meshi et al., 2016). For the purposes of this other study, participants filled out a scale measuring the degree of sharing of self-related information on social media (Carpenter, 2012). In addition, they completed the Mehrabian Conformity Scale (Mehrabian \& Stefl, 1995), the Narcissistic Personality Inventory (16 questions; Ames, Rose, \& Anderson, 2006), and the Rosenberg Self-Esteem Scale (Rosenberg, 1965). We focused our analyses on social desirability because of our specific hypotheses described above.

\section{MR image acquisition}

Scanning was performed at Freie Universität Berlin, Germany, using a 12-channel head coil on a 3T Siemens Trio scanner (Siemens Healthcare Diagnostics GmbH, Erlangen, Germany). Diffusion-weighted images were acquired using echo-planar imaging with the following parameters: 61 diffusion gradient directions; $b$-value $=1000 \mathrm{~s} / \mathrm{mm}^{2}$; $2 \times 2 \times 2 \mathrm{~mm}^{3}$ voxels; 69 axial slices; field of view $=208 \mathrm{~mm}$ $\times 208 \mathrm{~mm}$; repetition time $=10 \mathrm{~s}$; time echo $=94 \mathrm{~ms}$; bandwidth $1602 \mathrm{~Hz} / \mathrm{Px}$; fat saturation with a flip angle of $110^{\circ}$. The described sequence was repeated in two successive runs with one $b=0$ and 61 diffusion-weighted images collected per run. Acquisition lasted for $11 \mathrm{~min} 40$ seconds in total. 


\section{DTI analysis}

Voxel-wise statistical analysis was carried out using TractBased Spatial Statistics (TBSS) (S. M. Smith et al., 2006) in FMRIB's software library (FSL) (S. M. Smith et al., 2004). To note, TBSS is the leading technique for voxel-wise DTI analysis, but it is not without its limitations (for review, please see Bach et al., 2014). First, the two runs were concatenated and each of the volumes was affine registered to the $T_{2}$-weighted $b$ $=0$ volume of the second run using FLIRT (Jenkinson \& Smith, 2001). The fMRIB Diffusion Toolbox (FDT, https:// fsl.fmrib.ox.ac.uk/fslwiki/fdt) was used to correct the DTI data for head movement and residual eddy-current distortions present in the diffusion-weighted images. Each volume's B matrix was reoriented by applying the transformation matrix from the motion correction procedure. The nonbrain tissue was deleted from the image using FSL's brain extraction tool (S. M. Smith, 2002). Fractional anisotropy, eigenvector, and eigenvalue maps were computed. FA volumes were transformed into a common space and skeletonized as employed in Tract-Based Spatial Statistics (S. M. Smith et al., 2006). FA images were nonlinearly registered to the FMRIB58_FA template using the FNIRT tool (Andersson, Jenkinson, \& Smith, 2007). A mean FA image was generated and thinned (threshold at FA $>0.2$ ) to produce a mean FA skeleton representing the centers of tracts common to all participants. Finally, each subject's spatially normalized FA data were projected onto this common skeleton, and the resulting data fed into voxel-wise crosssubject statistics.

To investigate which diffusion component contributes to any observed FA differences, axial diffusivity (diffusivity along the axons) and radial diffusivity (diffusivity perpendicular to the axons) maps were created for each subject. Lower radial diffusivity values are associated with increased myelination, whereas axial diffusivity values are indicators of the degree of axonal coherence. Axial diffusivity was defined as the largest eigenvalue ( $\lambda 1)$, radial diffusivity as the mean of the second and third eigenvalues $([\lambda 2+\lambda 3] / 2)$ (Alexander, Lee, Lazar, \& Field, 2007). Axial and radial diffusivity maps were calculated using eigenvalue maps created by dtifit in FSL. Transformations derived from aligning FA images to the FMRIB58_FA template were applied to each subject's axial and radial diffusivity images. Projection vectors used to project each subject's FA data onto the group mean tract skeleton were also used to project each subject's axial and radial diffusivity data onto the tract skeleton.

To test the hypothesized positive relationship between social desirability and FA in external capsule, we carried out a region of interest (ROI) analysis. The external capsule ROI was defined as the overlap between (1) the anatomical location mask provided within the JHU ICBM-DTI- 81 WhiteMatter Labels Atlas (Mori et al., 2008) and (2) the group mean tract skeleton. Separate ROIs were extracted for left and right hemispheres. Social desirability scores were correlated with average FA values within the two ROIs across participants, controlling for age and gender using partial correlation. To ascertain if this analysis is appropriate given the size of our sample, we performed a power analysis. Based on previous research relating personality and DTI measures in predefined ROIs, we expected a correlation of around 0.45 (see, for example Baur, Hänggi, Langer, \& Jäncke, 2013; Chavez \& Heatherton, 2015; Eden et al., 2015). Aiming for power of above 0.80 , and a Bonferroni adjusted $p$ threshold $(p<$ .025 ), our power analysis indicates that a sample size of above 33 is appropriate for performing the suggested analysis. The sample size in our current study is 35 .

For our secondary analyses, each subject's axial and radial diffusivity within the bilateral external capsule ROIs were extracted from significant voxels using the fslmeants function of FSL. Pearson correlations between these mean values and social desirability scores were assessed. The purpose of these correlation analyses was to disentangle which of the diffusion components contributes to changes in the FA measure.

Whole-brain analysis of DTI data was carried out using nonparametric permutation-based inference (Nichols \& Holmes, 2002) as implemented in the Randomize tool in FSL (Winkler, Ridgway, Webster, Smith, \& Nichols, 2014). Voxel-wise whole-brain analyses were performed to assess the correlations between social desirability and values of FA within the previously extracted skeleton. Participants' gender and age were used as covariates. Threshold-free cluster enhancement (TFCE; S. M. Smith \& Nichols, 2009) was used for statistical inference. One thousand permutations were performed for each contrast. Statistical $p$-value maps were thresholded at $p<.033$, corrected for multiple comparisons. This strict threshold was used because analysis at $p<.05$ yielded results in one large cluster. We increased our $p$ threshold to break apart this cluster and discern results in different regions. The JHU ICBM-DTI-81 White-Matter Labels Atlas was used to identify the anatomical location of significant clusters in the brain (Mori et al., 2008; Mori, Oishi, \& Faria, 2009).

\section{Results}

\section{Social desirability}

Social desirability did not violate normality assumptions (Shapiro-Wilk statistic $=.961, p=.247)$. Age and gender did not correlate with social desirability, age: $t(33)=.302, p$ $=.764$; gender: $t(33)=.302, p=.764$. This is congruent with previous research that shows no effects (Loo \& Loewen, 2004; Reynolds, 1982) or very small effects of gender and age (Hebert et al., 1997; Ones et al., 1996). Internal consistency reliability of the questionnaire was calculated using KuderRichardson Formula 20 (Kuder \& Richardson, 1937), 
resulting in an alpha coefficient $(\alpha \mathrm{K}-\mathrm{R} 20=.66)$. Current alpha is slightly lower than the level at which the scale was validated $(\alpha \mathrm{K}-\mathrm{R} 20=.76)$ (Reynolds, 1982), which could be due to our sample size being insufficiently large for a reliable calculation of alpha (see Charter, 2003).

\section{External capsule ROI analysis}

Social desirability was found to positively correlate with FA values within the external capsule. Two separate partial correlations were performed for left and right external capsule ROIs using a significance threshold corrected for multiple comparisons ( $p<.025$; see Fig. 1), and controlling for age and gender. Both regions were significant (left: $r=.493, p=.002$; right: $r$ $=.353, p=.022$ ), and the effect sizes were within the range commonly found in the literature (Baur et al., 2013; Chavez \& Heatherton, 2015; Eden et al., 2015). Post hoc power analyses revealed .85 for the left and .54 for the right external capsule.

\section{Secondary external capsule ROI analyses}

We conducted further analyses to ascertain which diffusion components account for the observed FA results. Axial and radial diffusivity means within the bilateral external capsule were correlated with social desirability scores across participants. This analysis revealed a negative association between social desirability and radial diffusivity $(r=.438, p<.01)$. This result indicates an increased diffusivity perpendicular to axons of the above-mentioned tracts in participants with lower social desirability scores, and vice versa. No significant relationship was observed between axial diffusivity and social desirability $(r=.082, p=.615)$.

\section{Whole-brain DTI analysis}

FA values within four widely distributed clusters were found to positively correlate with social desirability scores $(p<.05$, corrected; see Fig. 2 and Table 1). First, a positive correlation with social desirability was observed in the external capsule. The external capsule conveys fibers to the caudate nucleus and putamen from the ventral and medial prefrontal cortex, ventral premotor cortex, precentral gyrus, rostral superior temporal region, and the inferotemporal and preoccipital regions (Schmahmann \& Pandya, 2009). Moreover, our results include significant voxels in the corona radiata, which contains fibers from both the internal and external capsule, and also connects the striatum with other regions of the brain such as the prefrontal cortex and thalamus (Mori et al., 2008; Schmahmann \& Pandya, 2009). We also observed a positive correlation with social desirability in the inferior frontooccipital fasciculus. To note, although the peak of this cluster appears to be in the inferior fronto-occipital fasciculus, several voxels in this cluster, while existing on the white-matter skeleton, are outside the JHU white-matter brain atlas. The inferior fronto-occipital fasciculus is a long-association fiber pathway that connects the occipital cortex, temporo-basal areas, and superior parietal lobe to the frontal lobe (Martino, Brogna, Robles, Vergani, \& Duffau, 2010). Lastly, our results include significant voxels all along the corpus callosum, which is a major interhemispheric commissural fiber connecting most of the neocortical areas (Schmahmann \& Pandya, 2009).

Regarding age and gender, our analysis revealed no significant clusters after correcting for multiple comparisons. To note, previous studies have found results with these two variables (Hsu et al., 2008). Our lack of results may be attributed to a relatively narrow range of participant ages and a stricter threshold when correcting for multiple comparisons. a
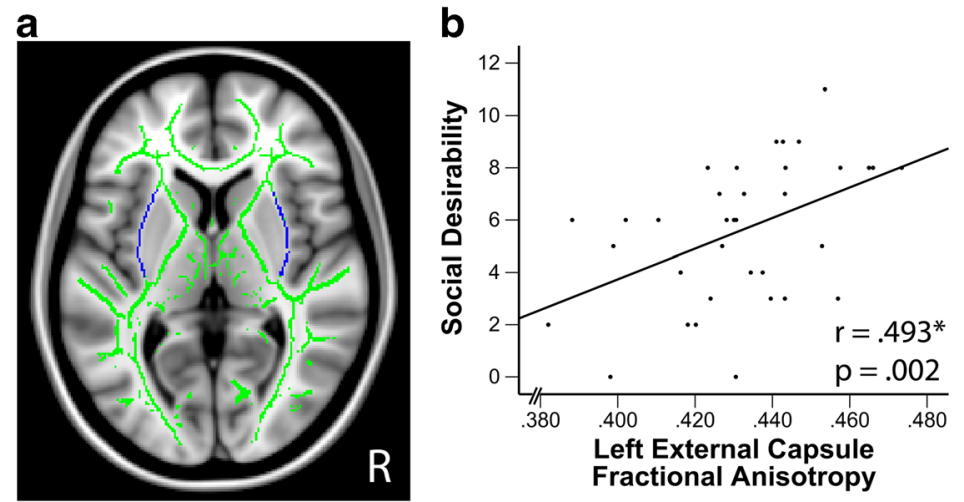

C

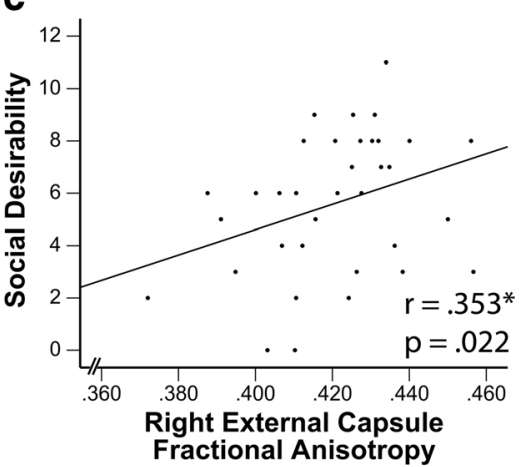

Fig. 1 Correlation analyses demonstrate the positive relationship between FA in the external capsule and social desirability. (a) The external capsule ROI's shown in blue, superimposed on the MNI template brain (coronal slice: $\mathrm{z}=5$ ). To note, these ROI masks were defined by the overlap between the group mean white-matter skeleton (indicated in green), and the external capsule as defined by the JHU
ICBM-DTI-81 White-Matter Labels Atlas (Mori et al., 2008). (b) Scatterplot showing correlation between FA in the left external capsule and social desirability. (c) Scatterplot showing correlation between FA in the right external capsule and social desirability (significance threshold corrected for multiple comparisons $(\mathrm{p}<0.025))$ 


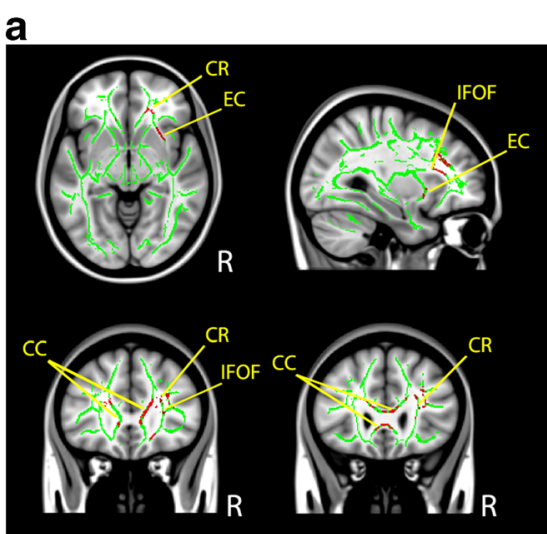

Fig. 2 Whole-brain associations between FA and social desirability. (a) Clusters where FA was significantly correlated with social desirability are indicated in red. Clockwise from top-left: coronal slice: $z=-6$; right sagittal slice: $\mathrm{x}=18$; coronal slice: $\mathrm{y}=31$; axial slice: $\mathrm{y}=26$. Results are presented on the MNI template brain, and images are presented in radiological convention. The group mean skeleton,

\section{Discussion}

Behaving in a socially desirable manner is crucially important for successful social functioning in our society. Based on previous neuroimaging research, we hypothesized a positive relationship between social desirability and FA in the external capsule. To test our hypothesis, we obtained diffusion tensor images of participants and examined the association between external capsule FA and social desirability, while controlling for age and gender. Our analyses revealed a significant positive correlation between social desirability and FA in both the left and right external capsule. Previous research on cognition and behaviors closely related to social desirability suggests that this behavior requires communication between a widely distributed network of brain regions, yet no experiment had previously investigated anatomical connectivity with respect to social desirability. Therefore, we examined associations between social desirability and FA indices within the whitematter skeleton of the whole brain, while controlling for age and gender. Whole-brain analysis revealed a significant positive relationship in several regions of the white-matter b

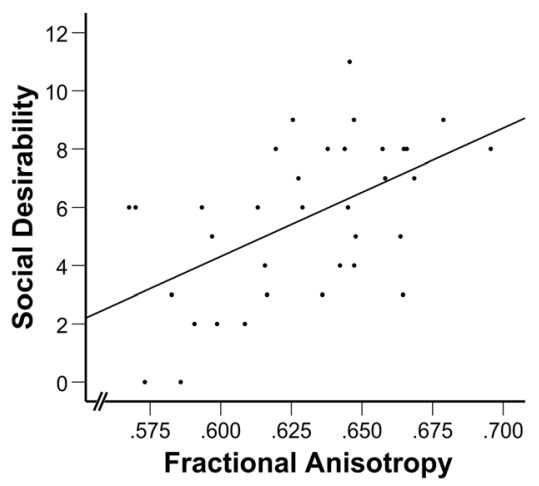

containing white-matter tracts common to all participants, is indicated in green. All displayed results are significant at $\mathrm{p}<0.05$ after correcting for FWE. (b) For illustration purposes, we plotted participants' social desirability scores against their mean fractional anisotropy values within the significant voxels. CC, corpus callosum; CR,corona radiata; EC, external capsule; IFOF, inferior fronto-occipital fasciculus

skeleton, such as the external capsule, corona radiata, corpus callosum, and inferior fronto-occipital fasciculus.

With regard to our result in the external capsule, this is a tract that relays fibers to the caudate nucleus and the putamen from the medial prefrontal cortex, ventral premotor cortex, precentral gyrus, rostral superior temporal region, and the inferotemporal and preoccipital regions (Schmahmann \& Pandya, 2009). We hypothesized that we would observe a positive relationship between social desirability and FA in the external capsule because of previous research on social desirability. Three previous studies found that social desirability is negatively associated with dopamine receptor availability in the striatum across participants (Cervenka et al., 2010; Huang et al., 2006; Reeves et al., 2007). In other words, the less dopamine signaling in the striatum of an individual, the more she behaves in a socially desirable manner. Furthermore, reduced dopamine signaling leads to an increase in whitematter integrity (Ebdrup et al., 2015; Thomason et al., 2010). Therefore, the positive relationship between social desirability and FA that we found is in line with these studies. In addition, behaving in a socially desirable manner involves

Table 1 Clusters where social desirability and fractional anisotropy were significantly correlated

\begin{tabular}{|c|c|c|c|c|c|c|c|c|c|}
\hline \multirow[t]{2}{*}{ Size $\left(\mathrm{mm}^{3}\right)$} & \multirow[t]{2}{*}{ FWE Corrected ( $P$ value) } & \multicolumn{3}{|c|}{ Peak } & \multicolumn{3}{|l|}{$\mathrm{COG}$} & \multirow[t]{2}{*}{ Hemi } & \multirow[t]{2}{*}{ Approximate tract location } \\
\hline & & $\mathrm{X}$ & $\mathrm{Y}$ & $\mathrm{Z}$ & $X$ & $\mathrm{Y}$ & $\mathrm{Z}$ & & \\
\hline 2,998 & .02 & 14 & 34 & -9 & 3.55 & 14.8 & 17.5 & $\mathrm{R}$ & $\mathrm{CR}, \mathrm{CC}$ \\
\hline 253 & .03 & 31 & 30 & 12 & 32.1 & 26.6 & 21.6 & $\mathrm{R}$ & IFOF \\
\hline 211 & .03 & 30 & 12 & -8 & 27.9 & 17.5 & -2.56 & $\mathrm{R}$ & EC \\
\hline 73 & .032 & 26 & 32 & 15 & 25.3 & 28.4 & 21.8 & $\mathrm{R}$ & $\mathrm{CR}$ \\
\hline
\end{tabular}

Note $\mathrm{FWE}=$ family-wise error rate $\mathrm{COG}=$ center of gravity; $\mathrm{Hemi}=$ hemisphere $\mathrm{EC}=$ external capsule $\mathrm{CR}=$ corona radiata $\mathrm{CC}=$ corpus callosum; IFOF $=$ inferior fronto-occipital fasciculus 
social reward processing, specifically when anticipating a desired outcome or evaluating if one's selected behavior achieved the desired outcome. Social rewards activate a network of brain regions including the ventral striatum and the medial prefrontal cortex (for review, see Fareri \& Delgado, 2014; Meshi, Tamir, \& Heekeren, 2015). For example, these regions are activated when a person receives cues that others understand her (Morelli, Torre, \& Eisenberger, 2013), agree with her (Campbell-Meiklejohn, Bach, Roepstorff, Dolan, \& Frith, 2010; Klucharev, Rijpkema, Smidts, Hytönen, \& Fernández, 2009; Meshi, Biele, Korn, \& Heekeren, 2012), like her (Davey et al., 2010), or think highly of her (Izuma et al., 2008; Korn et al., 2012; Meshi et al., 2013). Moreover, the computation of the value of social rewards relies on the connectivity between the striatum, ventromedial prefrontal cortex, and other brain regions (D. V. Smith et al., 2014). Speculatively, in order to compute the value of one's behavior in terms of social reward, the striatum likely receives signals from other brain regions that play a role in social processing (D. V. Smith et al., 2014; van den Bos, Talwar, \& McClure, 2013). If so, lower white-matter integrity could result in a less successful integration of social signals, which may reduce proneness to socially desirable behaviors. Our finding that social desirability is associated with the integrity of the external capsule fiber tract corroborates this view because the external capsule is a major pathway carrying information to the striatum, supporting the computation of social reward.

Our secondary analysis revealed that the association between social desirability and FA in the bilateral external capsule was mainly explained by radial diffusivity. Other studies demonstrating relationships between personality traits and FA have also found this pattern of correlations with radial diffusivity (Bjørnebekk, Westlye, Fjell, Grydeland, \& Walhovd, 2012; Xu \& Potenza, 2012). Radial diffusivity has been associated with myelination; decreased myelination results in increased freedom of cross-fiber diffusion in white matter, and thus an increase in radial diffusivity (Beaulieu, 2002; Burzynska et al., 2010; S. K. Song et al., 2002; S. K. Song et al., 2005; WheelerKingshott \& Cercignani, 2009). Our finding that radial diffusivity within the external capsule negatively correlates with social desirability points toward higher membrane integrity and myelination in the discussed tracts, and this could be a candidate mechanism underlying individual differences in social desirability. To note, however, diffusion tensor indices capture a complex plethora of physical tissue properties, and deriving conclusions about myelination, axonal density and membrane integrity should be taken with a grain of salt because they are likely an oversimplification of the data (Beaulieu, 2002; Wheeler-Kingshott \& Cercignani, 2009).

Our analysis also revealed that social desirability is positively associated with FA in the inferior fronto-occipital fasciculus, the corpus callosum, and the corona radiata. The inferior fronto-occipital fasciculus is a long-association fiber pathway that connects the occipital cortex, temporo-basal areas, and superior parietal lobe to the frontal lobe; the corpus callosum is an interhemispheric commissure that relays information between most of the cortical regions; and the corona radiata is a tract that connects the brain stem with most of the cortical regions (Martino et al., 2010; Schmahmann \& Pandya, 2009). Regarding the inferior fronto-occipital fasciculus, other individual differences studies with DTI measures have demonstrated associations across a broad range of neurocognitive functions, including attention, language, visual processing, and emotional empathy (Catani \& Thiebaut de Schotten, 2008; Duffau, Herbet, \& Moritz-Gasser, 2013; Parkinson \& Wheatley, 2014). In addition, our result in this area may also reflect the need for connectivity between the prefrontal cortex and the temporal and/or parietal lobes to perform mentalizing (Schurz et al., 2014) - mentalizing about the thoughts and interpretations of another person would be required when acting in a socially desirable manner. However, the lack of functional specificity of this tract makes it difficult to draw firm conclusions. Regarding the corpus callosum, our finding may reflect the need for cross-lateral connectivity of midline structures to perform self-related related cognition (Northoff et al., 2006) - self-related cognition would be required if individuals engage in self-deception during socially desirable behaviors, or if individuals simply consider how their behavior would impact themselves. Corpus callosum integrity has also been related to overall social functioning. This has been demonstrated through studies in both autism and brain lesion patients (Alexander, Lee, Lazar, Boudos, et al., 2007; Badaruddin et al., 2007; Beauchamp et al., 2009; Booth, Wallace, \& Happé, 2011; Paul et al., 2007; Symington, Paul, Symington, Ono, \& Brown, 2010). Our corpus callosum finding is in line with these studies; individuals with greater corpus callosum integrity demonstrate more trait social desirability. Other DTI studies have found correlations with personality traits and white-matter indices in the corpus callosum and corona radiata (see, for example, Bjørnebekk et al., 2012; Parkinson \& Wheatley, 2014; Xu \& Potenza, 2012); however, most studies refrain from discussing these findings. Findings observed in both the corpus callosum and corona radiata are difficult to interpret because the two tracts radiate out, reaching a vast number of cortical areas (Aboitiz, Scheibel, Fisher, \& Zaidel, 1992; Fitsiori, Nguyen, Karentzos, Delavelle, \& Vargas, 2011), and because current knowledge about these fiber tracts is mostly limited to motor functioning (see Jang, 2009; Kim \& Pope, 2005; Y. M. Song, 2007; Wahl \& Ziemann, 2008). Overall, with these broad regions, it is difficult to make specific interpretations linking the tracts to distinct types of cognition. This may be because a significant portion of the brain is involved in social cognition, with a variety of regions and networks computing and processing social behaviors (Stanley \& Adolphs, 2013). 
There are several points of discussion and possible limitations of our study that should be brought up. First, we do not claim that social reward processing explains socially desirable behavior entirely and exclusively but simply theorize that it is an important factor in the generation of this behavior. There are other types of cognition and processing that could be relevant for acting in a socially desirable manner, and we have discussed these above. Second, we did not include control behavioral measures. Therefore, we cannot absolutely determine behavioral specificity of our findings - ideally, researchers would be able to measure participants' actual, realworld amount of behaving in a socially desirable manner. Future research may be able to address this issue. Finally, although we theorize that there is a relationship between social desirability and reward processing, we are not able to provide evidence that social desirability correlates with performance in social reward processing. To our knowledge, there are no tasks that behaviorally measure performance in social reward tasks. Development of sensitive and reliable measurement tools for performance in social reward processing remains an interesting challenge for future research.

In conclusion, this study extends our understanding of the neural circuitry underlying behaving in a socially desirable manner. We present the first piece of evidence that socially desirable behavior correlates with white-matter microstructure of the external capsule, which supports connectivity between the prefrontal cortex and the striatum. This finding is in line with the idea that socially desirable behavior involves social reward processing. Our exploratory whole-brain analysis shows that social desirability also relies on connectivity between distributed brain regions involved in different types of cognitive processes.

Acknowledgements We would like to thank Loreen Mamerow for assistance during data collection.

Funding Funding for this research was provided by the Excellence Initiative of the German Research Foundation (DFG), "Languages of Emotion" [EXC302].

\section{Compliance with ethical standards}

Conflict of interest None declared.

\section{References}

Aboitiz, F., Scheibel, A. B., Fisher, R. S., \& Zaidel, E. (1992). Fiber composition of the human corpus callosum. Brain Research, 598(1/2), 143-153.

Alexander, A. L., Lee, J. E., Lazar, M., Boudos, R., DuBray, M. B., Oakes, T. R., ... Lainhart, J. E. (2007). Diffusion tensor imaging of the corpus callosum in Autism. NeuroImage, 34(1), 61-73.

Alexander, A. L., Lee, J. E., Lazar, M., \& Field, A. S. (2007). Diffusion tensor imaging of the brain. Neurotherapeutics, 4(3), 316-329.
Ames, D. R., Rose, P., \& Anderson, C. P. (2006). The NPI-16 as a short measure of narcissism. Journal of Research in Personality, 40(4), $440-450$.

Andersson, J. L. R., Jenkinson, M., \& Smith, S. (2007). Non-linear registration aka spatial normalisation (FMRIB Technial Report TR07JA2). Retrieved from https://www.fmrib.ox.ac.uk/datasets/ techrep/tr07ja2/tr07ja2.pdf.

Bach, M., Laun, F. B., Leemans, A., Tax, C. M. W., Biessels, G. J., Stieltjes, B., \& Maier-Hein, K. H. (2014). Methodological considerations on tract-based spatial statistics (TBSS). NeuroImage, 100, 358-369

Badaruddin, D. H., Andrews, G. L., Bölte, S., Schilmoeller, K. J., Schilmoeller, G., Paul, L. K., \& Brown, W. S. (2007). Social and behavioral problems of children with agenesis of the corpus callosum. Child Psychiatry and Human Development, 38(4), 287-302.

Baur, V., Hänggi, J., Langer, N., \& Jäncke, L. (2013). Resting-state functional and structural connectivity within an insula-amygdala route specifically index state and trait anxiety. Biological Psychiatry, 73(1), 85-92.

Beauchamp, M. H., Anderson, V. A., Catroppa, C., Maller, J. J., Godfrey, C., Rosenfeld, J. V., \& Kean, M. (2009). Implications of reduced callosal area for social skills after severe traumatic brain injury in children. Journal of Neurotrauma, 26(10), 1645-1654.

Beaulieu, C. (2002). The basis of anisotropic water diffusion in the nervous system - A technical review. NMR in Biomedicine, 15(7/8), 435-455.

Bjørnebekk, A., Westlye, L. T., Fjell, A. M., Grydeland, H., \& Walhovd, K. B. (2012). Social reward dependence and brain white-matter microstructure. Cerebral Cortex, 22(11), 2672-2679.

Booth, R., Wallace, G. L., \& Happé, F. (2011). Connectivity and the corpus callosum in autism spectrum conditions: Insights from comparison of autism and callosal agenesis. Progress in Brain Research, 189, 303-317.

Borkenau, P., \& Ostendorf, F. (1992). Social desirability scales as a moderator and suppressor variables. European Journal of Personality, 6(3), 199-214.

Borkenau, P., \& Zaltauskas, K. (2009). Effects of self-enhancement on agreement on personality profiles. European Journal of Personality, 23(2), 107-123.

Bou Malham, P., \& Saucier, G. (2016). The conceptual link between social desirability and cultural normativity. International Journal of Psychology, 51(6), 474-480.

Burzynska, A. Z., Preuschhof, C., Bäckman, L., Nyberg, L., Li, S. C., Lindenberger, U., \& Heekeren, H. R. (2010). Age-related differences in white-matter microstructure: Region-specific patterns of diffusivity. NeuroImage, 49(3), 2104-2112.

Campbell-Meiklejohn, D. K., Bach, D. R., Roepstorff, A., Dolan, R. J., \& Frith, C. D. (2010). How the opinion of others affects our valuation of objects. Current Biology, 20(13), 1165-1170.

Carpenter, C. J. (2012). Narcissism on Facebook: Self-promotional and anti-social behavior. Personality and Individual Differences, 52(4), 482-486.

Catani, M., \& Thiebaut de Schotten, M. (2008). A diffusion tensor imaging tractography atlas for virtual in vivo dissections. Cortex, 44(8), $1105-1132$.

Cervenka, S., Gustavsson, J. P., Halldin, C., \& Farde, L. (2010). Association between striatal and extrastriatal dopamine D2-receptor binding and social desirability. NeuroImage, 50(1), 323-328.

Charter, R. R. A. (2003). Study samples are too small to produce sufficiently precise reliability coefficients-ProQuest. The Journal of General Psychology, 130(2), 117-129.

Chavez, R. S., \& Heatherton, T. F. (2015). Multimodal frontostriatal connectivity underlies individual differences in self-esteem. Social Cognitive and Affective Neuroscience, 10(3), 364-370.

Crowne, D. P., \& Marlowe, D. (1960). A new scale of social desirability independent of psychopathology. Journal of Consulting Psychology, 24(4), 349-354. 
Davey, C. G., Allen, N. B., Harrison, B. J., Dwyer, D. B., \& Yücel, M. (2010). Being liked activates primary reward and midline selfrelated brain regions. Human Brain Mapping, 31(4), 660-668.

Diener, E., Sandvik, E., Pavot, W., \& Gallagher, D. (1991). Response artifacts in the measurement of subjective well-being. Social Indicators Research, 24(1), 35-56.

Duffau, H., Herbet, G., \& Moritz-Gasser, S. (2013). Toward a pluri-component, multimodal, and dynamic organization of the ventral semantic stream in humans: Lessons from stimulation mapping in awake patients. Frontiers in Systems Neuroscience, 7, 44.

Ebdrup, B. H., Raghava, J. M., Nielsen, M. Ø., Rostrup, E., \& Glenthøj, B. (2015). Frontal fasciculi and psychotic symptoms in antipsychotic-naive patients with schizophrenia before and after 6 weeks of selective dopamine D2/3 receptor blockade. Journal of Psychiatry and Neuroscience, 41(2), 133-141.

Eden, A. S., Schreiber, J., Anwander, A., Keuper, K., Laeger, I., Zwanzger, P., ... Dobel, C. (2015). Emotion regulation and trait anxiety are predicted by the microstructure of fibers between amygdala and prefrontal cortex. Journal of Neuroscience, 35(15), 6020-6027.

Edwards, A. L. (1957). The social desirability variable in personality assessment and research. New York: Dryden Press.

Fareri, D. S., \& Delgado, M. R. (2014). The importance of social rewards and social networks in the human brain. The Neuroscientist, 20(4), $387-402$.

Fitsiori, A., Nguyen, D., Karentzos, A., Delavelle, J., \& Vargas, M. I. (2011). The corpus callosum: White matter or terra incognita. British Journal of Radiology, 84(997), 5-18.

Hebert, J. R., Ma, Y., Clemow, L., Ockene, I. S., Saperia, G., Stanek, E. J., ... Ockene, J. K. (1997). Gender differences in social desirability and social approval bias in dietary self-report. American Journal of Epidemiology, 146(12), 1046-1055.

Holden, R. R. (2010). Social desirability. In I. B. Weiner \& W. E. Craighead (Eds.), The Corsini encyclopedia of psychology (4th). New York: Wiley. https://doi.org/10.1002/9780470479216.corpsy0889

Hsu, J.-L., Leemans, A., Bai, C.-H., Lee, C.-H., Tsai, Y.-F., Chiu, H.-C., \& Chen, W.-H. (2008). Gender differences and age-related white matter changes of the human brain: A diffusion tensor imaging study. NeuroImage, 39(2), 566-577.

Huang, C. L., Yang, Y. K., Chu, C. L., Lee, I. H., Yeh, T. L., Chen, P. S., \& Chiu, N. T. (2006). The association between the Lie scale of the Maudsley personality inventory and striatal dopamine D2/D3 receptor availability of healthy Chinese community subjects. European Psychiatry, 21(1), 62-65.

Ivanoff, A., \& Jang, S. J. (1991). The role of hopelessness and social desirability in predicting suicidal behavior: A study of prison inmates. Journal of Consulting and Clinical Psychology, 59(3), 394-399.

Izuma, K., Saito, D. N., \& Sadato, N. (2008). Processing of social and monetary rewards in the human striatum. Neuron, 58(2), 284-294.

Jang, S. H. (2009). A review of corticospinal tract location at corona radiata and posterior limb of the internal capsule in human brain. NeuroRehabilitation, 24(3), 279-283.

Jenkinson, M., \& Smith, S. (2001). A global optimisation method for robust affine registration of brain images. Medical Image Analysis, 5(2), 143-156.

Kim, J. S., \& Pope, A. (2005). Somatotopically located motor fibers in corona radiata: Evidence from subcortical small infarcts. Neurology, 64(8), 1438-1440.

Klucharev, V., Rijpkema, M., Smidts, A., Hytönen, K., \& Fernández, G. (2009). Reinforcement learning signal predicts social conformity. Neuron, 61(1), 140-151.

Knoch, D., Nitsche, M. A., Fischbacher, U., Eisenegger, C., PascualLeone, A., \& Fehr, E. (2008). Studying the neurobiology of social interaction with transcranial direct current stimulation-The example of punishing unfairness. Cerebral Cortex, 18(9), 1987-1990.
Korn, C. W., Prehn, K., Park, S. Q., Walter, H., \& Heekeren, H. R. (2012). Positively biased processing of self-relevant social feedback. The Journal of Neuroscience, 32(47), 16832-16844.

Kuder, G. F., \& Richardson, M. W. (1937). The theory of the estimation of test reliability. Psychometrika, 2(3), 151-160.

Lane, R. D., Merikangas, K. R., Schwartz, G. E., Huang, S. S., \& Prusoff, B. A. (1990). Inverse relationship between defensiveness and lifetime prevalence of psychiatric disorder. American Journal of Psychiatry, 147(5), 573-578.

Leite, W. L., \& Beretvas, N. S. (2005). Validation of scores on the Marlowe-Crowne Social Desirability Scale and the Balanced Inventory of Desirable Responding. Educational and Psychological Measurement, 65(1), 140-154.

Linehan, M. M., \& Nielsen, S. L. (1983). Social desirability: Its relevance to the measurement of hopelessness and suicidal behavior. Journal of Consulting and Clinical Psychology, 51(1), 141-143.

Loo, R., \& Loewen, P. (2004). Confirmatory factor analyses of scores from full and short versions of the Marlowe-Crowne Social Desirability Scale. Journal of Applied Social Psychology, 34(11), 2343-2352.

Loo, R., \& Thorpe, K. (2000). Confirmatory factor analyses of the full and short versions of the Marlowe-Crowne Social Desirability Scale. The Journal of Social Psychology, 140(5), 628-635.

Martino, J., Brogna, C., Robles, S. G., Vergani, F., \& Duffau, H. (2010). Anatomic dissection of the inferior fronto-occipital fasciculus revisited in the lights of brain stimulation data. Cortex, 46(5), 691-699.

McCrae, R. R., \& Costa, P. T. (1983). Social desirability scales: More substance than style. Journal of Consulting and Clinical Psychology, 51(6), 882-888.

Mehrabian, A., \& Stefl, C. A. (1995). Basic temperament components of loneliness, shyness, and conformity. Social Behavior and Personality, 23(3), 253-263.

Meshi, D., Biele, G., Korn, C. W., \& Heekeren, H. R. (2012). How expert advice influences decision making. PLOS ONE, 7(11), e49748.

Meshi, D., Mamerow, L., Kirilina, E., Morawetz, C., Margulies, D. S., \& Heekeren, H. R. (2016). Sharing self-related information is associated with intrinsic functional connectivity of cortical midline brain regions. Scientific Reports, 6, 22491. https://doi.org/10.1038/srep22491

Meshi, D., Morawetz, C., \& Heekeren, H. R. (2013). Nucleus accumbens response to gains in reputation for the self relative to gains for others predicts social media use. Frontiers in Human Neuroscience, 7, 439. https://doi.org/10.3389/fnhum.2013.00439

Meshi, D., Tamir, D. I., \& Heekeren, H. R. (2015). The emerging neuroscience of social media. Trends in Cognitive Sciences, 19(12), 771782.

Mills, J. F., \& Kroner, D. G. (2005). An investigation into the relationship between socially desirable responding and offender self-report. Psychological Services, 2(1), 70-80.

Miotto, P., \& Preti, A. (2008). Suicide ideation and social desirability among school-aged young people. Journal of Adolescence, 31(4), 519-533.

Morelli, S. A., Torre, J. B., \& Eisenberger, N. I. (2013). The neural bases of feeling understood and not understood. Social Cognitive and Affective Neuroscience, 9(12), 1890-1896.

Mori, S., Oishi, K., \& Faria, A. V. (2009). White matter atlases based on diffusion tensor imaging. Current Opinion in Neurology, 22(4), 362-369.

Mori, S., Oishi, K., Jiang, H., Jiang, L., Li, X., Akhter, K., ... Mazziotta, J. (2008). Stereotaxic white matter atlas based on diffusion tensor imaging in an ICBM template. NeuroImage, 40(2), 570-582.

Nichols, T. E., \& Holmes, A. P. (2002). Nonparametric permutation tests for functional neuroimaging: A primer with examples. Human Brain Mapping, 15(1), 1-25.

Northoff, G., Heinzel, A., de Greck, M., Bermpohl, F., Dobrowolny, H., \& Panksepp, J. (2006). Self-referential processing in our brain-A metaanalysis of imaging studies on the self. NeuroImage, 31(1), 440-457. 
Ones, D. S., Viswesvaran, C., \& Reiss, A. D. (1996). Role of social desirability in personality testing for personnel selection: The red herring. Journal of Applied Psychology, 81(6), 660-679.

Parkinson, C., \& Wheatley, T. (2014). Relating anatomical and social connectivity: White-matter microstructure predicts emotional empathy. Cerebral Cortex, 24(3), 614-625.

Paul, L. K., Brown, W. S., Adolphs, R., Tyszka, J. M., Richards, L. J., Mukherjee, P., \& Sherr, E. H. (2007). Agenesis of the corpus callosum: genetic, developmental and functional aspects of connectivity. Nature Reviews Neuroscience, 8(4), 287-299.

Paulhus, D. L. (1984). Two-component models of socially desirable responding. Journal of Personality and Social Psychology, 46(3), 598-609.

Pauls, C. A., \& Stemmler, G. (2003). Substance and bias in social desirability responding. Personality and Individual Differences, 35(2), 263-275.

Reeves, S. J., Mehta, M. A., Montgomery, A. J., Amiras, D., Egerton, A., Howard, R. J., \& Grasby, P. M. (2007). Striatal dopamine (D2) receptor availability predicts socially desirable responding. NeuroImage, 34(4), 1782-1789.

Reynolds, W. M. (1982). Development of reliable and valid short forms of the Marlowe-Crowne Social Desirability Scale. Journal of Clinical Psychology, 38(1), 119-126.

Rilling, J. K., \& Sanfey, A. G. (2011). The neuroscience of social decision making. Annual Review of Psychology, 62, 23-48.

Robinette, R. L. (1991). The relationship between the Marlowe-Crowne Form C and the validity scales of the MMPI. Journal of Clinical Psychology, 47(3), 396-399.

Rosenberg, M. (1965). Society and the adolescent self-image. Princeton: Princeton University Press.

Ruff, C. C., \& Fehr, E. (2014). The neurobiology of rewards and values in social decision making. Nature Reviews Neuroscience, 15(8), 549-562.

Russell, R. J. H., \& Wells, P. A. (1992). Social desirability and quality of marriage. Personality and Individual Differences, 13(7), 787-791.

Schmahmann, J. D., \& Pandya, D. N. (2009). Fiber pathways of the brain. New York: Oxford University Press.

Schurz, M., Radua, J., Aichhorn, M., Richlan, F., \& Perner, J. (2014). Fractionating theory of mind: A meta-analysis of functional brain imaging studies. Neuroscience and Biobehavioral Reviews, 42, 9-34.

Smith, D. V., Clithero, J. A., Boltuck, S. E., \& Huettel, S. A. (2014). Functional connectivity with ventromedial prefrontal cortex reflects subjective value for social rewards. Social Cognitive and Affective Neuroscience, 9(12), 2017-2025.

Smith, S. M. (2002). Fast robust automated brain extraction. Human Brain Mapping, 17(3), 143-155.

Smith, S. M., Jenkinson, M., Johansen-Berg, H., Rueckert, D., Nichols, T. E., Mackay, C. E., ... Matthews, P. M. (2006). Tract-based spatial statistics: Voxelwise analysis of multi-subject diffusion data. NeuroImage, 31(4), 1487-1505.

Smith, S. M., Jenkinson, M., Woolrich, M. W., Beckmann, C. F., Behrens, T. E. J., Johansen-Berg, H., ... Matthews, P. M. (2004).
Advances in functional and structural MR image analysis and implementation as FSL. NeuroImage, 23, S208-S219.

Smith, S. M., \& Nichols, T. E. (2009). Threshold-free cluster enhancement: Addressing problems of smoothing, threshold dependence and localisation in cluster inference. NeuroImage, 44(1), 83-98.

Song, S. K., Sun, S.-W., Ramsbottom, M. J., Chang, C., Russell, J., \& Cross, A. H. (2002). Dysmyelination revealed through MRI as increased radial (but unchanged axial) diffusion of water. NeuroImage, 17(3), 1429-1436.

Song, S. K., Yoshino, J., Le, T. Q., Lin, S. J., Sun, S. W., Cross, A. H., \& Armstrong, R. C. (2005). Demyelination increases radial diffusivity in corpus callosum of mouse brain. NeuroImage, 26(1), 132-140.

Song, Y. M. (2007). Somatotopic organization of motor fibers in the corona radiata in monoparetic patients with small subcortical infarct. Stroke, 38(8), 2353-2355.

Spitzer, M., Fischbacher, U., Herrnberger, B., Grön, G., \& Fehr, E. (2007). The neural signature of social norm compliance. Neuron, 56(1), 185-196.

Stanley, D. A., \& Adolphs, R. (2013). Toward a neural basis for social behavior. Neuron, 80(3), 816-826.

Symington, S. H., Paul, L. K., Symington, M. F., Ono, M., \& Brown, W. S. (2010). Social cognition in individuals with agenesis of the corpus callosum. Social Neuroscience, 5(3), 296-308.

Tan, L., \& Grace, R. C. (2008). Social desirability and sexual offenders: A review. Sexual Abuse: A Journal of Research and Treatment, 20(1), 61-87.

Thomason, M. E., Dougherty, R. F., Colich, N. L., Perry, L. M., Rykhlevskaia, E. I., Louro, H. M., ... Gotlib, I. H. (2010). COMT genotype affects prefrontal white matter pathways in children and adolescents. NeuroImage, 53(3), 926-934.

Twenge, J. M., \& Im, C. (2007). Changes in the need for social approval, 1958-2001. Journal of Research in Personality, 41(1), 171-189.

Uziel, L. (2010). Rethinking social desirability scales: From impression management to interpersonally oriented self-control. Perspectives on Psychological Science, 5(3), 243-262.

van den Bos, W., Talwar, A., \& McClure, S. M. (2013). Neural correlates of reinforcement learning and social preferences in competitive bidding. The Journal of Neuroscience, 33(5), 2137-2146.

Wahl, M., \& Ziemann, U. (2008). The human motor corpus callosum. Reviews in the Neurosciences, 19(6), 451-466.

Wheeler-Kingshott, C. A. M., \& Cercignani, M. (2009). About "axial" and "radial" diffusivities. Magnetic Resonance in Medicine, 61(5), $1255-1260$.

Winkler, A. M., Ridgway, G. R., Webster, M. A., Smith, S. M., \& Nichols, T. E. (2014). Permutation inference for the general linear model. NeuroImage, 92, 381-397.

Xu, J., \& Potenza, M. N. (2012). White matter integrity and five-factor personality measures in healthy adults. NeuroImage, 59(1), 800-807.

Zook, A. I., \& Sipps, G. J. (1985). Cross-validation of a short form of the Marlowe-Crowne Social Desirability Scale. Journal of Clinical Psychology, 41(2), 236-238. 